\title{
Unrecognized News from the Filter Model and Review of the Spence Model
}

\author{
Monem Abidi \\ VPNC Research Laboratory N-O, FSJEG of Jendouba, University of Jendouba, Tunisia
}

\begin{abstract}
The objective of this article is to cross a line of reflection which makes it possible to trace more solid legibility in what is similar to the explanation of the choices of educational investments and the regulation of the labor market. This analysis aims to propose a new approach which adopts as a reference the role of aptitude in determining the future of the labor market, in terms of individual decisions to invest in training and terms of expected productivity about of the signal issued by the diploma.The methodology mainly refers to the signal theory and filter theory. In our study, first, we will integrate a new concept to better analyze the different forms of filter (productive and nonproductive) on which collective productivity, individual productivity, and profitability largely depend. Second, we reformulate Spence's model by integrating two levels of discrimination and three groups. This breakdown becomes more complex, but it allows a better understanding of the choices, the gains, as well as the balance on the labor market.The re-examination of the filter theory exposes the idea of regularizing the university and market system close to the abilities of individuals. The demonstrations also show that the signal effect does not own its assets, it depends on other signals. The results of this treaty are applicable in terms of rationalizing the policies of funds intended for training, guiding the functioning of the labor market as well as tackling the problem of unemployment.
\end{abstract}

Codes JEL: E24, E32, G12, J23.

Keywords: Filter; signal; gains; balance; labor market; diploma

DOI: $10.7176 / \mathrm{JESD} / 11-16-21$

Publication date:August $31^{\text {st }} 2020$

\section{Introduction}

For decades the trajectory of educational investment (private and public) has been strongly guided by theories of human capital (HC) of signal and filter. Spreading a lot of anchor of development of analysis and criticism but, their secrets remains fierce to any domestication.

The present analysis hopes to find other lines of thought and to trace a little-known field of study, thus offering a new passage in what approaches the explanation of the choices of educational investments.

The central assumption of the $\mathrm{HC}$ model is that education is recognized as the key determinant of the structure and evolution of individual incomes, where individuals, by forgoing an immediate gain that they would receive if they entered the labor market immediately, hope to increase their productive capacities and the associated market value.

From this premise The HC theory believes that education transmits knowledge that will be transformed into skills, they will increase the productive efficiency of the individual and in its strongest version HC theory judges that knowledge are necessary. This justifies the higher remuneration of graduates, insofar as this knowledge allows higher productivity.

This nomenclature changes the behavior of the labor supply in the sense that the most qualified individuals are willing to extend their studies and therefore consciously bear additional costs and a longer period of inactivity, hoping to be compensated in the future. .

This construction only takes place if the two partners (company and employer) are well satisfied and their strategies are approved by the labor market.

The HC theory was subject you to in s critical s by many economic theories that have tried to identify additional clarification to the issue of productivity in the company and the mechanisms of the labor market.

The foundation stone was laid by Arrow (1973) and Spence (1973).

Spence believes that in a market where information is imperfect, it is impossible for employers to know the productivity of the individuals he will recruit. He therefore uses the signal issued by the diplomas, and according to his past experience, the employer anticipates the level of productivity and therefore the level of remuneration.

It is in this sense that the study of signals aims to obtain information or make it meaningful (Kunt, 1999) through two aspects of study: the emission and reception of the signal. The signal is considered to be "intentionally sent to one or more targeted receptors that are likely to be in a position to hear the signal" (Spence, 2002).

Even more, with Arrow (1973), education has no direct effect on productivity; it only gives a label to the individual to say that he is supposed to be more productive. In this sense, education is only a filter and does not add value to the individual.

On the other side Thurow (1975) considers in his model of competition for employment that workers are only productive if it is allowed in other words, it is no longer workers who are productive, but it is rather the job they 
hold. This job, and for it to be profitable, requires specific training, which is supposed to be easier for those who have a diploma.

Therefore, education only sorts out individuals who are able to better design this specific training and therefore, the cost will be lower for educated individuals.

The company then targets these individuals and compensates them to fill jobs requiring significant internal training in the hope of achieving higher productivity.

Another radical approach proposed by Gintis (1971) which stipulates that education builds in individuals attitudes of order and obedience, which will be demanded and valued by the employer.

Piore and Doeringer (1971) propose the segmentation of the labor market theory, which claims that in a segmented market we find workers with similar productive characteristics, but who are paid differently, which calls into question this mechanical relationship between productivity and remuneration, especially when there is no mobility between sectors.

The parchment theory of Berg (1971) considers two groups of the same skills, but they differ in obtaining degrees or not, he finds that the rate of return is the same, which shows that this is no longer the diploma which ensures profitability and therefore the filter, but it is rather the registration in the sector.

The works of Jarousse and Mingat (1986) constitute the pillars, in what approaches confirmation of the signal or filter phenomenon. By specifying a model based on the number of years of standardized studies and the position of the individual within his generation, shows his empirical robustness in the interpretation of income distribution.

More recent studies have tried to dissect the filter and signal effect, by identifying the reaction of the labor market and incorporating characteristics specific to its functioning.

Olivier Chariot (2005), by modeling interactions between education and participation in the labor market, demonstrates that education has not only a positive effect on workers' wage earnings, but also a beneficial effect on their duration of employability.

Arjen Edzes , Marije Hamersma , Viktor Venhorst, Jouke van Dijk (2015) find that regional factors have a significant impact on the decision to continue studying and on the chances of obtaining a job.

Claude Diebolt, Magali Jaoul - Grammare (2016), demonstrate that individual choices are made by giving particular importance to the number of places and the gain in their choice, but also by trying to anticipate the behavior of others.

Isabelle Lebon, Therese Rebiere (2016), believe that a government seeking to maximize employment should base the funding of higher education on the productivity gap between educated and uneducated workers.

Ariel J. Binder and John Bound, (2019) explain that the decline in labor market activity is linked to the drop in wages of men who only had a college diploma. This calls into question the filter effect as a factor generating collective well-being and regulating the labor market.

Mauricio Benegas, Marcio Veras Correâ (2020), and by studying the link between the policies of distortion of the school offer and the performances of the labor market, shows that the policies of open enrollment and school equity have ambiguous effects in the labor market.

All these advances, which are sometimes complementary and contradictory in other cases, reduce the relevance of putting forward theories that are based solely on costs and rent to explain individual education decisions.

The reformulation of the model begins with the determination of the framework which delimits this relationship between companies and holders of diplomas. The novelty of our reformulation respects the framework of signal theory, and tries to answer the questions of contemporary society, in particular with regard to the rapid increase in unemployment of graduates, and in particular the possibility of building a kind of balance. between the different constituents of the labor market.

The article is organized as follows :

Section I deals with the general presentation of the reporting process, where the decisions of employers will be taken according to the signal, and similarly for investors in signal the decisions will be guided by their valuations on the labor market.

Section II examines the non-productive filter, where marginal productivity manifests itself only by innate ability, which will allow distinguishing the parameters that guide decisions under the pretext that education only reveals skills.

Section III discusses the productive filter, where marginal productivity depends on both ability and level of education. It turns out that the decomposition shows that those with higher aptitudes will seek the highest signal.

Section IV completes the study with a reformulation of the Spence model, distinguishing two levels of discrimination and three groups of individuals.

This demarcation makes it possible to compare the position of different categories according to different parameters and to sketch the separating balance.

The study is decisive with regard to the equilibrium on the labor market, the determination of wages, the investment decisions the creation and the destruction of equilibrium separator and mixer and the transfer of benefits. 


\section{The reporting process}

The Spence model assumes that individuals are differentiated by their "p" productivities. And the population is divided into two groups, with productivity " $p_{1}$ " and " $p_{2}$ " with $p_{1}<p_{2}$. The proportion of the two groups in the population is " $q_{1}$ " for the first group and " $q_{2}$ " for the second.

At first, and in a situation of imperfect information, the salary will be the same for all individuals and it will equalize the average productivity of the population:

$\bar{p}=p_{1} q_{1}+p_{2}\left(1-q_{1}\right)$

This equation penalizes the most able in favor of the less able, and their loss will be:

$\bar{p}-p_{2}=-q_{1}\left(p_{2}-p_{1}\right) \prec 0$

This loss increases if the proportion of the least able $\left(q_{1}\right)$ increases in the population or $\left(p_{2}-p_{1}\right)$ becomes more important, that is to say that, the crack in terms of productivity increases between the two categories. .

On the other hand, the less able realize a surplus of:

$\bar{p}-p_{1}=\left(1-q_{1}\right)\left(p_{2}-p_{1}\right) \succ 0$

This surplus increases with the decrease of " $q_{1}$ " or the growth of $\left(p_{2}-p_{1}\right)$.

As a result, the signal process appears as a necessary phenomenon because it accords with human nature, which seeks to be distinguished. So workers will invest rationally in training so that employers can detect their skills and pay them based on those skills. It is in this sense that the diploma is considered as a signal.

For employers, they will use their previous experiences and data on the labor market, to enhance these signals and will be paid according to this basis.

The strategy of a worker therefore consists in choosing the optimum level of signaling which allows him to acquire the greatest income. Knowing that signal equilibrium assumes that there is a negative correlation between signal cost and skill.

We consider " $Y$ " the level of training that ensures discrimination, the cost of education will be in the form:

$C_{i}\left(Y, p_{i}\right)=\frac{Y}{p_{i}} \quad$ With $i=1$ or 2 .

From this structure, we see that the less able will pay more to have the same level of education, ie " $\bar{Y}$ " such that:

$$
\frac{p_{2}-p_{1}}{p_{1}} \prec \bar{Y} \prec \frac{p_{2}-p_{1}}{p_{2}} \quad \rightarrow \quad p_{2}-p_{1} \prec p_{1} \bar{Y} \quad \text { and } \quad p_{2}-p_{1} \succ p_{2} \bar{Y}
$$

And since skills are unobservable, employers' decisions will be made based on the " $Y$ " signal.

$w(Y / Y<\bar{Y})=E(p / Y<\bar{Y})=p_{1}$ proportion "ql" with probability "l".

$w(Y / Y \geq \bar{Y})=E(p / Y \geq \bar{Y})=p_{2}$ proportion " $q_{2}$ " with probability "l".

The possibilities offered for an individual are either " $Y=0$ " or " $Y=\bar{Y}$ ", since then the decision of individuals of the type " $p_{I}$ " is then " $Y=0$ " and receive a salary equal to " $p_{I}$ ", since the cost here is zero, and they will not choose " $Y=\bar{Y}$ " because their income will be:

$p_{2}-\left(\bar{Y} / p_{l}\right)$ which is next $[\mathrm{A}]$ less than " $p_{I}$ ".

For individuals of type " $p_{2}$ ", they will choose " $Y=\bar{Y}$ " which gives them an income equal to " $p_{2}-\left(\bar{Y} / p_{2}\right)$ " which is according to $[\mathrm{A}]$ greater than " $p_{2}$ ". If the decision is " $Y=0$ " the income will be " $p_{1}$ " which is not optimal. If we combine the two optimal choices we will have: $p_{1}<\bar{Y}<p_{2}$.

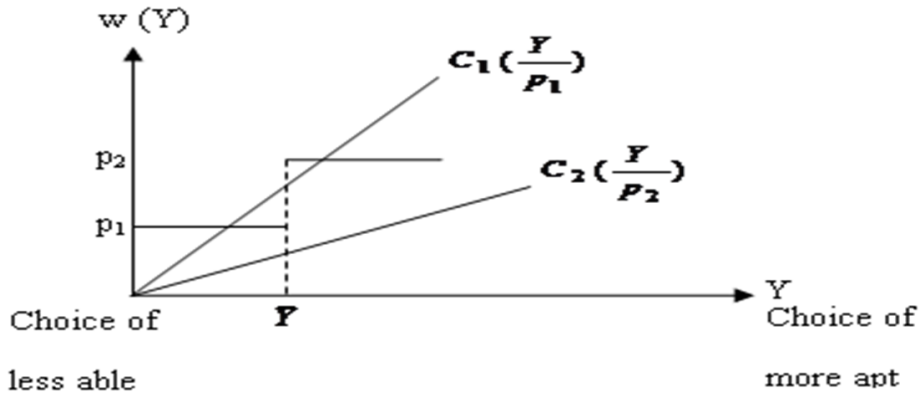

Figure 1: Optimal choice of reporting

However, for an edifying analysis we must distinguish at least three types of filters: the non-productive filter with these two perfect and imperfect forms and the productive filter. 


\section{The perfect non-productive filter}

2.1 General framework of the model

The main idea is that education brings nothing in terms of production; its role is limited to the emission of the signal while always adopting the hypothesis that the cost of education and the ability are correlated negative, so marginal productivity manifests itself only by the innate ability " $a$ ".

Where " $Y$ " is the level of education acquired.

The salary is only remunerated at the base of " $Y$ " because " $a$ " is unobservable, $w=w(Y)$, as well as the cost $C$ (a, $Y)=Y / a$, because the cost is negatively correlated with the skills so that the net income to be maximized is:

$R(a, Y)=w(Y)-C(a, Y)$

$$
=w(Y)-\frac{Y}{a}
$$

The reporting balance presupposes two conditions: the first is, the rationality of employers demanding as well as $w(Y(a))=\operatorname{Pm}(a, Y)=a$, the second reflects the maximization of income for individuals. We will therefore have:

$R^{\prime}(a, Y)=0$ and $R^{\prime \prime}(a, Y) \prec 0$

$\rightarrow R^{\prime}(a, Y)=0 \rightarrow w^{\prime}(Y)-C^{\prime}(a, Y)=0$

$\rightarrow w^{\prime}(Y)=\frac{1}{a}$ and $w^{\prime \prime}(Y) \prec 0$

$\rightarrow w^{\prime}(Y)=\frac{1}{a} \rightarrow a=\frac{1}{w^{\prime}(Y)}$

Now we have:

$w(Y)=a \rightarrow w(y)=\frac{1}{w^{\prime}(Y)}$. This amounts to solving a differential equation, at equilibrium we will have:

$w^{*}(Y, k)=(2 Y+k)^{\frac{1}{2}}$ with "k" an integration constant.

However according to [B], we have:

$$
w^{* \prime}(Y)-C^{\prime}(a, Y)=0 \quad \rightarrow(2 Y+k)^{-\frac{1}{2}}-\frac{1}{a}=0 \quad \rightarrow Y^{*}(a)=\frac{a^{2}-k}{2}
$$

Then $w^{*}$ will be:

$$
w^{*}\left[Y^{*}(a)\right]=\left[2\left(\frac{a^{2}-k}{2}\right)+k\right]^{\frac{1}{2}}=a=\operatorname{Pm}(a)
$$

And

$$
\begin{aligned}
R^{*}\left(a, Y^{*}\right)= & a-\frac{Y^{*}}{a}=a-\frac{a^{2}-k}{2 a} \\
= & \frac{2 a^{2}-a^{2}+k}{2 a} \\
& =\frac{a^{2}+k}{2 a}=\frac{a}{2}+\frac{k}{2 a}
\end{aligned}
$$

To see the impact of the skill on the demand for diplomas, we will consider double the first skill, i.e. " $2 a$ ". We will have $C=\left(Y^{\prime} / 2 a\right)$ where " $Y^{\prime \prime}$ the new level of study knowing that the skill is equal to " $2 a^{\prime \prime}$ :

$$
\begin{aligned}
& R\left(2 a, Y^{\prime}\right)=w\left(Y^{\prime}\right)-C\left(2 a, Y^{\prime}\right) \\
& R^{\prime}\left(2 a, Y^{\prime}\right)=0 \\
& \rightarrow w^{\prime}\left(Y^{\prime}\right)=C^{\prime}\left(2 a, Y^{\prime}\right)=\frac{1}{2 a}
\end{aligned}
$$

Similarly by replacing " $2 a$ ", we will have a differential equation whose resolution is also the same: 
$w^{*}\left(Y^{\prime}, k\right)=\left(2 Y^{\prime}+k\right)^{\frac{1}{2}}$

[D]

By introducing $[\mathrm{D}]$ in $[\mathrm{C}]$, we can write:

$\left(2 Y^{\prime}+k\right)^{-\frac{1}{2}}-\frac{1}{2 a}=0 \quad \rightarrow Y^{\prime *}(a)=\frac{4 a^{2}-k}{2}$

Hence:

$$
\frac{Y^{\prime^{*}}}{Y^{*}}=\frac{4 a^{2}-k}{2} \times \frac{2}{a^{2}-k}=\frac{4 a^{2}-k}{a^{2}-k} \succ \frac{4 a^{2}-4 k}{a^{2}-k}=4
$$

It is noted that there is a hypersensitivity to the ability: if the ability is doubled, the level of education will be multiplied by more than " 4 ".

This conception is strictly in favor of the fittest. However, this formulation was based on very strong assumptions, assuming that there is a perfect match between skills, marginal productivity and diploma, and also canceling out market imperfections.

\subsection{The imperfect non-productive filter}

For this model, the main difference is manifested in the insemination of the cost function, where it now depends on " $a$ " and " $\theta$ ". The latter indicates funding opportunities, therefore:

$C=C(a, \theta, Y)=Y /(a+\theta)$.

The salary offered will also depend on the expected conditional productivity of the " $Y$ " signal. And by proceeding to the same approach of income maximization, we will have:

$$
Y^{*}(a, \theta)=\left(\frac{a+\theta}{2}\right)^{2} ; w^{*}(a, \theta)=\frac{a+\theta}{2} ; R^{*}(a, \theta)=\frac{a+\theta}{4}
$$

If we want to detect the effect of aptitude on investment in training, we double the aptitude. We will therefore have:

$$
Y^{\prime *}(2 a, \theta)=\left(\frac{2 a+\theta}{2}\right)^{2}
$$

Hence:

$$
\frac{Y^{\prime^{*}}}{Y^{*}}=\left(\frac{2 a+\theta}{2}\right)^{2} \times\left(\frac{2}{a+\theta}\right)^{2}=\left(\frac{2 a+\theta}{a+\theta}\right)^{2} \prec 4
$$

Compared to the previous model, the introduction of the variable " $\theta$ " (funding opportunity) slows investment in training.

The introduction of the variable " $\theta$ " also implies a process of compensation between the latter and the variable " $a$ ", where there will be a tendency to find the most suitable individuals, but who are deprived of financial opportunities at the same level education than individuals who have strong funding opportunities, but weaker skills.

Regarding compensation, it is " $w=(a+\theta) / 2$ ", but productivity or skill is " $a$ ".

For employers to be satisfied, the salary must be at least " $a$ ":

$w=a=(a+\theta) / 2$.

$$
\rightarrow a-(a+\theta) / 2=0 \rightarrow(a-\theta)=0 \rightarrow a=\theta
$$

If "w>a $\rightarrow \theta>a$ ": that is to say that the funding opportunities in the population exceed skills. In this case, we will see a second regulation of wages by employers until arriving at " $w=a "$.

If " $w<a \rightarrow \theta<a$ ": indicates that the skills found in the population exceed the funding opportunities. In this case, individuals will try to invest further in education so that employers can better detect their skills and reduce " $w$ " to "a".

\section{The productive filter}

\subsection{Presentation of the model}

For this model, it is considered that education does not reveal innate skills only, but has its own productivity, and the cost depends only on ability $(C=C(a, Y)=Y / a)$. Marginal productivity depends on both ability and level of education. The specification of the model is as follows:

$$
\operatorname{Pm}=\operatorname{Pm}(a, Y)=a Y^{\alpha} \quad \text { where } 0<\alpha<1
$$

This specificity describes that, the elasticity of productivity with respect to the level of education " $Y$ " is equal to the salary " $w(Y) "$, thus the individual will maximize his income: 


$$
\begin{aligned}
R(a, Y)= & w(Y)-C(a, Y) \\
& =w(Y)-\frac{Y}{a}
\end{aligned}
$$

3.2. Determination of balance:

When looking for " $Y^{*}$ ", the first order maximization conditions are:

$w^{\prime}(Y)=1 / a$ and $w^{\prime \prime}(Y)<0$

Since:

$$
w=P m=a Y^{\alpha} \rightarrow a=\frac{w(Y)}{Y^{\alpha}} \rightarrow w^{\prime}(Y)=\frac{Y^{\alpha}}{w(Y)} \quad \rightarrow w^{\prime}(Y) \times w(Y)-Y^{\alpha}=0
$$

It is a differential equation whose solution is:

$$
w^{*}(Y)=\left[\frac{2 Y^{1+\alpha}}{1+\alpha}\right]^{\frac{1}{2}} \quad(\text { With the integration constant } k=0 \text { ) }
$$

There fore:

$$
Y^{*}(a)=\left[\frac{a^{2}(1+\alpha)}{2}\right]^{\frac{1}{1-\alpha}}
$$

And

$$
\begin{aligned}
R^{*}(a, Y)= & w^{*}(Y)-\frac{Y^{*}}{a}=a\left(Y^{*}\right)^{\alpha}-\frac{Y^{*}}{a}=\frac{Y^{*}}{a}\left[a^{2}\left(Y^{*}\right)^{\alpha-1}-1\right] \\
& =\frac{Y^{*}}{a}\left(\frac{2}{1+\alpha}-1\right)=\left[\frac{a^{2}}{2}(1+\alpha)\right]^{\frac{1}{1-\alpha}}\left[\frac{(1-\alpha)}{a(1+\alpha)}\right] \\
& =\left[\frac{a^{1+\alpha}}{2}(1+\alpha)\right]^{\frac{1}{1-\alpha}}\left[\frac{1-\alpha}{1+\alpha}\right] \\
& =\left[\frac{a^{1+\alpha}}{2}(1+\alpha)^{\alpha}\right]^{\frac{1}{1-\alpha}}(1-\alpha) \\
& =\left(\frac{a}{2}\right)^{\frac{1}{1-\alpha}}[a(1+\alpha)]^{\frac{\alpha}{1-\alpha}}(1-\alpha)
\end{aligned}
$$

If we want to look for the impact of the skill on education, we will double the initial skill and see its effect on " $Y$ ". So we will have:

$$
\begin{aligned}
& Y^{*}(2 a)=2 a^{2}(1+\alpha)^{\frac{1}{1-\alpha}} \\
& \frac{Y^{\prime}(2 a)}{Y(a)}=\left[\frac{2 a^{2}(1+\alpha)}{a^{2}(1+\alpha)} \times 2\right]^{\frac{1}{1-\alpha}} \\
& \rightarrow \quad=(4)^{\frac{1}{1-\alpha}}=\left(2^{2}\right)^{\frac{1}{1-\alpha}} \succ 1
\end{aligned}
$$

This result clearly shows that those with higher abilities will seek the highest signal. For the study of the general framework we consider " $\lambda a "$, with $\lambda>1$ : 


$$
\begin{aligned}
& \mathrm{Y}^{*}(\lambda a)=\left[\frac{\lambda^{2} a^{2}(1+\alpha)}{2}\right]^{\frac{1}{1-\alpha}} \\
& \quad \frac{Y^{\prime}(\lambda a)}{Y(a)}=\left[\lambda^{2}\right]^{\frac{1}{1-\alpha}}
\end{aligned}
$$

We will examine this signal demand as a function of " $\alpha$ " (the elasticity of productivity with respect to education level " $Y ")$.

$\rightarrow$ If " $\alpha$ " tends to " 0 " (that is, we tend to cancel the effect of education):

$\frac{Y^{\prime}(\lambda a)}{Y(a)}=\lambda^{2}$ signal demand is reduced to skill supplement (demand for higher signals)

$\rightarrow$ If " $\alpha$ " tends to " $1 ":$

$\frac{Y^{\prime}(\lambda a)}{Y(a)} \rightarrow \infty$, There is no demand limit for the highest signals.

Similarly, we can calculate the ratios of net equilibrium income:

$$
\begin{aligned}
\frac{R^{\prime}(\lambda a, Y)}{R(a, Y)} & =\frac{\lambda^{\frac{1+\alpha}{1-\alpha}}\left[\frac{(1+\alpha) a^{2}}{2}\right]^{\frac{1}{1-\alpha}}\left[\frac{(1-\alpha)}{a(1+\alpha)}\right]}{\left[\frac{(1+\alpha) a^{2}}{2}\right]^{\frac{1}{1-\alpha}}\left[\frac{(1-\alpha)}{a(1+\alpha)}\right]} \\
& =\lambda^{\frac{1+\alpha}{1-\alpha}}
\end{aligned}
$$

Likewise if " $\alpha \rightarrow 0$ " therefore $\frac{R^{\prime}(\lambda a, Y)}{R(a, Y)} \rightarrow \lambda:$ The difference in net income is reduced only to the difference in ability.

If " $\alpha \rightarrow l$ " then $\frac{R^{\prime}(\lambda a, Y)}{R(a, Y)} \rightarrow \infty$ : There will be a very large depreciation of the income of the least able.

\subsection{The filter hypothesis and jobs held}

Another interesting advanced vision, insofar as it allows comparing the two theories, of $\mathrm{HC}$ and of filter for the same formulation through the introduction of another dimension which is the influence of the occupied job where variables are defined as follows:

Schooling "s" to obtain a level of diploma " $Y^{\prime \prime}: s=s(a, Y)$, with $s_{a}<0$ : the duration of schooling is a function decreasing with ability.

$s_{Y}>0$ : the duration of studies varies in the same direction as the level of education for the same skills.

$s_{a Y}<0$ : the marginal increase in education is obtained more easily for the most able.

Productivity depends on " $a$ " and " $Y$ ". So: $P=P(a, Y)$

With $P_{a}, P_{Y} \geq 0$, meaning that productivity varies positively with ability and education.

The salary is a function of " $Y^{\prime \prime}: w=w(Y)$.

Income discounted over a life cycle is expressed as follows:

$R(a, Y, w)=e^{-r s(a, Y)} w^{1-\alpha}(Y) P^{\alpha}(a, Y)$, with $0 \leq \alpha \leq 1$.

The equilibrium is obtained by the maximization of the income and a rational anticipation of the employers, i.e. looking for " $Y(a)$ " which maximizes $R(a, Y, w(Y))$ such that $w(Y(a))=P(a, Y(a))^{l}$.

We will now study the earnings profiles according to the nature of the job. We will start with the first pole where

\footnotetext{
${ }^{1}$ We assume that: $P_{Y Y}<0, \quad s_{Y Y}>0, \quad d T M S_{P a} / d a<0$.
} 
jobs require a filter $(\alpha \rightarrow 0)$ while admitting that employers' forecasts are not biased:

$\widetilde{P}=P(a, Y) \widetilde{\varepsilon} \quad$ With $E(\widetilde{\varepsilon})=1$

So the expected income is:

$$
\begin{aligned}
& R(a, Y, w)=e^{-r s(a, Y)} w(Y)^{1-\alpha} E(\widetilde{P})^{\alpha} \\
& =e^{-r s(a, Y)} w(Y)^{1-\alpha} P(a, Y)^{\alpha} E(\widetilde{\varepsilon})^{\alpha} \\
& =e^{-r s(a, Y)} w(Y)^{1-\alpha} P(a, Y)^{\alpha} \\
& \rightarrow \log R(a, Y, w)=(1-\alpha) \log w(Y)+\alpha \log P(a, Y)-r s(a, Y)
\end{aligned}
$$

We ask:

$$
\operatorname{Iog} w(Y)=W(Y) \text { et } \log P(a, Y)=p(a, Y)
$$

Hence the maximization of the logarithm of the income allows us to give us the optimal level of signal " $Y$ " knowing the ability " $a$ ":

$$
\underset{Y}{\operatorname{Max}} \log R(a, Y, w)=\underset{Y}{\operatorname{Max}}[(1-\alpha) W(Y)+\alpha p(a, Y)-r s(a, Y)]
$$

First and second order conditions:

$(1-\alpha) W^{\prime}(Y)+\alpha p_{Y}(a, Y)-r s_{Y}(a, Y)=0 \quad Y \succ 0$

And

$(1-\alpha) W^{\prime \prime}(Y)+\alpha p_{Y Y}-r s_{Y Y}(a, Y) \leq 0$

Which shows that the optimal investment corresponds to the equalization between the rate of return of a signal $\left[(1-\alpha) W^{\prime}(Y)+\alpha p_{Y}(a, Y)\right]$, and its marginal cost $r s_{Y}(a, Y)$. The solution is represented on the figure

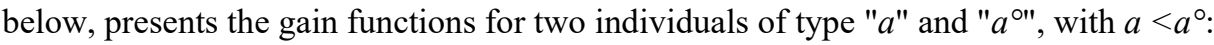

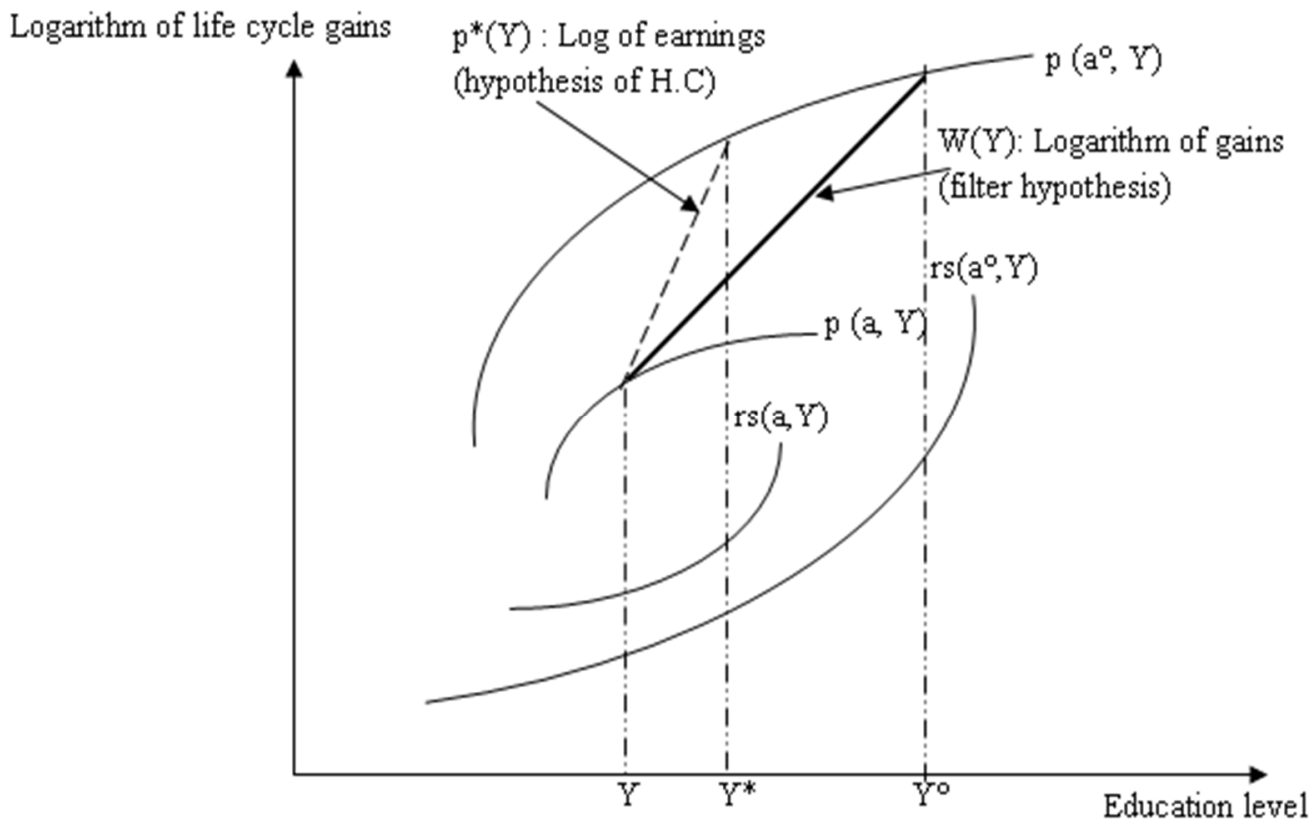

Figure 2: Gain functions over a life cycle

Note that the balance for the less able $(a)$ is the same in both cases. Whereas for the most apt individuals $\left(a^{\circ}\right)$, they will adapt to a higher signal equilibrium including the filter conditions than $\mathrm{HC}$, translating a straight line of the gain profiles, flatter for the filter process $(W(Y))$ than for the process without filter $\left(p^{*}(Y)\right)$.

It remains for us to study the second axis, that is to say that concerning jobs that are not subject to the filter $(\alpha \rightarrow 1)$, which requires another hypothesis, assuming that there is a logic compensation between jobs, but at the same income for the same signal level. So, for a job not subject to the filter, the income is:

$R(a, \hat{Y})=e^{-r s(a, \hat{Y})} \widehat{P}(a, \hat{Y})$

This income is assumed to be the same for another job. 
$\rightarrow e^{-r s(a, \widehat{Y})} \hat{P}(a, \hat{Y})=e^{-r s(a, Y)} w(Y)^{1-\alpha} P(a, Y)^{\alpha}$

Let's ask:

$\widehat{p}(a, \widehat{Y})=\log \hat{P}(a, \hat{Y})$

$\rightarrow \hat{p}(a, \hat{Y})-r s(a, \hat{Y})=(1-\alpha) W(Y)+\alpha p(a, Y)-r s(a, Y)$

The optimum is chosen such that:

$(1-\alpha) W^{\prime}(Y)+\alpha p_{Y}(a, Y)=r s_{Y}(a, Y)$

And since the return on education "W' $(\mathrm{Y})$ " exceeds private income, this balance allows us to write:

$p_{Y}(a, Y) \prec r s_{Y}(a, Y)$

We have: $p_{Y}(a, Y) \geq \hat{p}_{Y}(a, Y)^{1}$

So, we will have: $\hat{p}_{Y}(a, Y) \prec r s_{Y}(a, Y)$

While " $\hat{Y}(a)$ " program solution (without filter): $\hat{p}_{Y}(a, \hat{Y})-r s_{Y}(a, \hat{Y})=0$

And taking into account the hypotheses already mentioned $\left(P_{Y}<0\right.$ et $\left.s_{Y}>0\right)$, we will have: $\hat{Y}(a) \prec Y(a)$

From these analytical developments, we draw the following conclusion: individuals in the unfiltered sector spend less time in school than those in the filtered sector. However, the income distributed in the unfiltered sector is lower than that distributed in the filtered sector. However, these income supplements are very small (flattened slope).

It should be noted here that the majority of these results are very sensitive, and that they largely depend on the basic formulation which is based on strong assumptions, so all these results are likely to be demolished if we change the benchmarks a bit.

\section{Reformulation of the Spence Model}

\subsection{Basic principle}

Spence's (1973) model is based on a basic assumption which assumes that individuals are unequal in the face of signaling costs, since ability reduces costs. Spence considered two productivity groups " $A$ " and " $B$ " " 1 " and "2", with $(1<2)$. Group " $A$ " has a cost " $y$ " and group " $B$ " a cost " $y / 2$ " (with better skills), to acquire the level of training " $y *$ paid at their marginal productivity. That is to say, " 1 " for group " $A$ ", and "2" for group " $B "$.

Spence has shown that group " $A$ " has an interest in choosing a level of training " $y=0$ " and group " $B$ " a level of training" $y^{* "}$. At signaling equilibrium, it is conceivable that the situation of group " $A$ " will deteriorate, while the situation of group " $B$ " will become more favorable than if the workforce of group " $A$ " is greater than " $50 \%$ ". That is to say, the size of group " $B$ " must be a minority in the population.

Our goal is to extrapolate the illustration of Spence from "2" to "3" groups $(A, B$ and $C)$, and from a level of discrimination " $y *$ " to two levels " $x * "$ and " $y * "$.

This extrapolation is very delicate, very important because it can highlight two points already overlooked so far:

- It illustrates the possible choices and the relative gains of an average diploma.

- It allows highlighting three choices instead of two: medium signaling, strong signaling, and no signaling.

Suppose that there are three groups of workers in the labor market " $q_{1}, q_{2}, q_{3}$ " respectively, and productivity groups "1, 2 and 4", respectively. The training is distinguished by an index " $y$ " (the signaling means), which the agents can have by bearing a cost inversely proportional to their skills:

- For group " $A$ ", the acquisition cost of a level " $y$ " is exactly " $y$ " units.

- For group " $B$ ", the acquisition cost of a level " $y$ " is exactly " $y / 2$ " units.

- For group " $C$ ", the acquisition cost of a level " $y$ " is exactly " $y / 4$ " units.

Similarly:

- For group " $A$ ", the acquisition cost of a level " $x$ " is exactly " $x$ " units.

- For group " $B$ ", the acquisition cost of a level " $x$ " is exactly " $x / 2$ " units.

- For group " $C$ ", the acquisition cost of a level " $x$ " is exactly " $x / 4$ " units.

This is in line with the basic assumption of cost reduction with skills (we assume here that " $y=2 x "$ ).

For the employer, there are now two levels of training discrimination: the first $\left(x^{*}\right)$, below which it is certain to recruit a person of marginal productivity equal to " 1 ". The second $\left(y^{*}\right)$, below which it is certain to recruit a person of marginal productivity equal to twice the first, that is to say "2". Beyond that, he can estimate a marginal productivity equal to twice the second, ie "4". With " $y^{*}=2 x^{* "}$ which expresses that the signaling " $y^{* "}$ is twice the signaling of " $x * 12$, the same for costs and marginal productivity. Next, we will explain the training strategy adopted by the candidates according to their abilities.

\footnotetext{
${ }^{1}$ The productivity of a filtered job is greater than another unfiltered one.

${ }^{2}$ Individuals must follow the hierarchy to move from one level to another.
} 
Group $A: c=y / 2\left(\right.$ level $\left.x^{*}\right)$

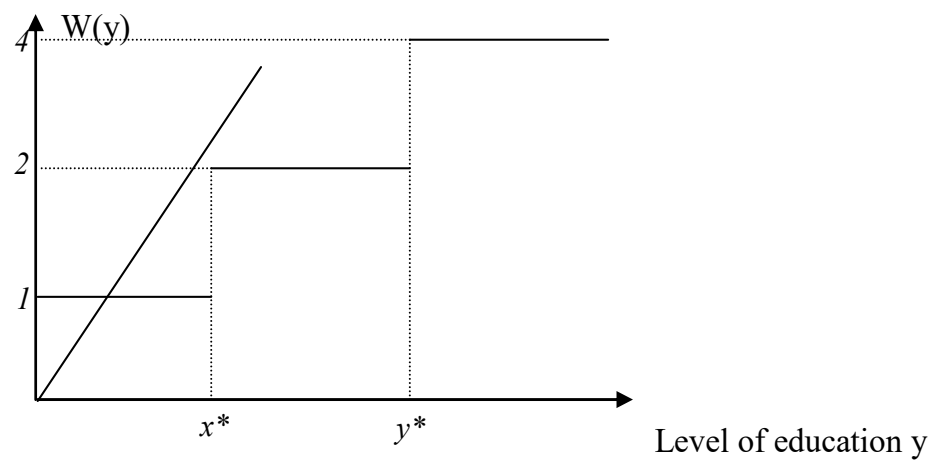

0 : optimal choice of training for group A individuals

$$
1>\left(2-y^{*} / 2\right)
$$

Group $B: c=y / 4\left(\right.$ level $\left.x^{*}\right)$

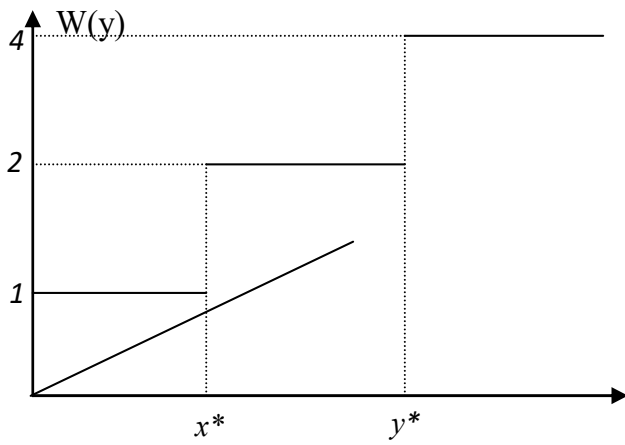

Level of education y

$x^{*}$ : optimal choice of training for group $B$ individuals

$(2-y * / 4)>1$ and $2>(4-y * / 2)$

Group $C: \mathrm{c}=\mathrm{y} / 4($ level $y *)$

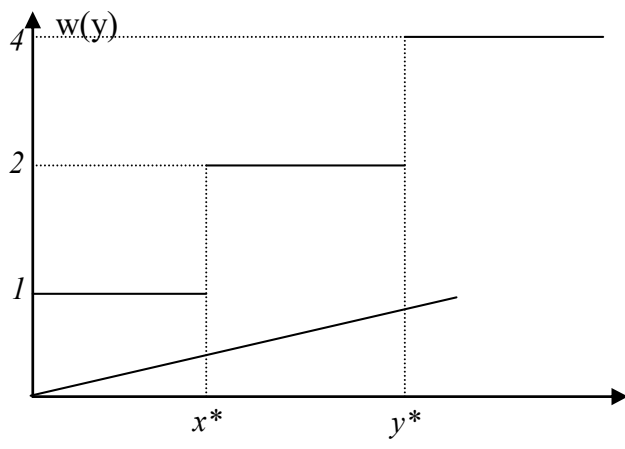

Level of education y

$y^{*}$ : optimal choice of training for individuals in group $C$ with $\left(4-y^{*} / 4\right)>2$

Figure 3: Optimal reporting choices (three groups) ${ }^{l}$

Reading the graph shows that group " $A$ " must support twice what group " $B$ " must support, to have the same signal level " $x * "$. Likewise, group " $B$ " must pay double what group " $C$ " pays to have the same signal level " $y^{* "}$. We end up with three cases:

- Achieve a level of training " $y=y^{* "}($ group $C)$.

- Achieving a level of training " $y=y^{* / 2}=x^{* \prime \prime}$ (group $B, y^{*}$ does nothing for this group).

- Renounce all training " $y=0$ " (group " $A$ ", " $x * "$ does not yield anything for this group as does " $y * ")$.

- For group " $A$ ", as long as additional training does not yield anything, that is to say that the people in this group

\footnotetext{
${ }^{1}$ Source: the author
} 
always have " $1>\left(2-y^{*} / 2\right) "$, they will choose " $y=0 "$.

- For group " $B$ ", individuals have an interest in reaching " $y=y^{*} / 2=x^{*}$, since for them " $\left(2-y^{* / 4}\right)>1$ ", and not accessing additional training since it costs more than it pays, and they have "2>(4-y*/2)".

- For group $C$, its people have an interest in reaching " $y=y^{* "}$, since this level brings them more than its cost, and they have "(4-y*/4)>2".

It is easy to see that the following condition must be met:

$1<x *<2$ and $2<y^{*}<4$.

These equilibrium thus found, must be compared to the signaling equilibrium by the diploma which is obtained by the following principle: let " $q_{1}, q_{2}, q_{3}$ " be the probabilities of recruiting respectively an individual from group " $A$ ", from the group " $B$ " and group " $C$ ", and these individuals are assumed to be paid at their marginal productivity. So, we will have:

$1 . q_{1}+2 . q_{2}+4 . q_{3}$

Now we know that: $q_{1}+q_{2}+q_{3}=1$

So: $q_{3}=1-q_{1}-q_{2}$

Hence: $1 . q_{1}+2 . q_{2}+4 .\left(1-q_{1}-q_{2}\right)=4-\left(3 . q_{1}+2 \cdot q_{2}\right)$

The latter equality represents signaling balance.

We notice that, the less able group " $A$ " is penalized by this signaling equilibrium, if we know that its remuneration $(1)$ is always lower than this equilibrium whatever " $q_{1}$ and $q_{2} "$.

For group " $B$ ", and for it to benefit from this balance, its remuneration (2) must be greater than this balance: $2>4-\left(3 . q_{1}+2 . q_{2}\right)$

Hence: $-\left(3 . q_{1}+2 . q_{2}\right)<-2$ and $3 . q_{1}+2 . q_{2}>2(\mathbf{I})$

And we know that we can write the previous balance in another way:

$1-q_{2}-q_{3}+2 q_{2}+4 q_{3}=1+q_{2}+3 \cdot q_{3}$

So: $2>1+q_{2}+3 . q_{3}$

Hence: $q_{2}+3 . q_{3}<1$

At the end: $-2 . q_{2}-6 . q_{3}>-2$ (II)

And if we sum the two results (I) and (II) we will have: $3 . q_{1}-6 . q_{3}>0$

Hence: $q_{1}>2 . q_{3}$

Therefore, individuals with high-level qualifications must be in the minority, and their workforce must not exceed half of the workforce of the least qualified individuals.

As a result, group " $B$ " can only benefit from this balance if the size of group " $C$ " is reduced. It is for this reason that the assessment of the diploma of this class does not depend directly on itself (on their own signal), but on the class which is superior in terms of level of training. In other words, if the diplomas of the upper class depreciate, the group of the lower class will be automatically penalized, and with more serious consequences.

For individuals in group " $C$ ", it seems that they are winners in this balance, but they can only benefit from the "4$y^{*} / 4$ " remuneration, when their number is low on the market.

\subsection{Costs and strategies}

We extend this model in order to clarify the phenomenon of depreciation, by relying on signaling costs and the two frontiers of discrimination that we have already established and we combine them with university policy. Various scenarios appear.

It is now assumed that the acquisition costs of the different training levels are perfectly divisible and that the acquisition cost of a training unit for group " $A$ " is " $a_{1}$ ", " $a_{2}$ " for the group " $B$ " and " $a_{3}$ " for group " $C$ ", and since the productivity of the agents is in decreasing relation with the costs, we can write that " $a_{3}<a_{2}<a_{1}$ ".

For group " $B$ " and " $C$ " to benefit from signaling balance, the following conditions must be fulfilled:

$2-a_{21}>2-a_{11}$

$2-a_{31}>2-a_{11}$

$2-a_{31}>2-a_{21}$

$4-a_{22}>4-a_{12}$

$4-a_{32}>4-a_{22}$

$4-a_{32}>4-a_{12}$

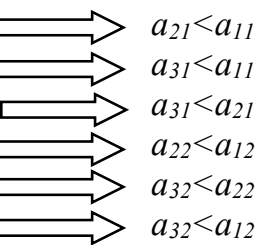

The first index indicates the group $(A: 1, B: 2$ and $C: 3)$ and the second index shows the level of discrimination " $x$ *" designated by " 1 " and " $y *$ "designated by " 2 ".

We can show that:

$a_{32}<a_{22}<a_{12}$ and $a_{31}<a_{21}<a_{12}$ (I).

This means that the transition to level " $y *$ is less expensive for group " $B$ " compared to group " $A$ " and even less for group " $C$ ", and likewise for level " $x *$ ".

But, the problem which arises at this level is the comparison between " $a_{21}$ and $a_{31}$ " on the one hand, and between " $a_{11}$ ", " $a_{22}$ ", on the other hand, so as to reveal their meaning in terms of decisions. Four cases to analyze: 
First case: $a_{21}>a_{32}$ and $a_{11}>a_{22}$ :

$a_{21}>a_{32}$ : implies that reaching level " $y^{* "}$ by group " $C$ " is easier than reaching level " $x^{* "}$ by group " $B "$ ". We will therefore have an increase in the size of group " $C$ ", since once beyond the level " $x *$ ", reaching level " $y * "$ is easier, and part of group " $B$ " fetch the level " $y *$ (if conditions are favorable), although its ability is relatively reduced to grab this level.

$a_{11}>a_{22}$ : reaching level " $y * "$ is easier for group " $B$ " relative to the effort provided by group " $A$ ", to reach level " $x * "$. If we follow the same reasoning, we will have an increase in the size of group " $C$ ".

Therefore, this description provides suitable conditions for individuals to reach the highest level " $y *$ (situation in favor of the most able, group " $B$ " and " $C$ ").

Second case: $a_{21}<a_{32}$ and $a_{11}>a_{22}$ :

$a_{21}<a_{32}$ : reaching level " $x * "$ by group " $B$ " is easier than reaching level " $y^{* \prime}$ by group " $C$ ". So, part of the group " $C$ " will be dissuaded (however, their skills allow them to move to a higher level), and will decide the level $" x * "$ (if the conditions are not favorable). A concentration will therefore be located in group " $B "$.

$a_{11}<a_{22}$ : massification of group " $C$ ".

In total, this case favors the less able to the detriment of the more able.

Third case: $a_{21}<a_{32}$ and $a_{11}<a_{22}$ :

$a_{21}<a_{32}$ : massification of group " $B$ ".

$a_{11}<a_{22}$ : reaching level " $x *$ " is easier for group " $A$ " than reaching " $y^{* 1}$ for group " $B$ ", and there we will have an increase in staff from group " $B$ ".

What makes a concentration in group " $B$ ".

Fourth case: $a_{21}>a_{32}$ and $a_{11}<a_{22}$ :

$a_{21}>a_{32}$ : massification of group " $C$ ".

$a_{11}<a_{22}$ : massification of group " $B$ ".

In this case, we will have an increase in levels, but at the expense of group " $B$ ".

Thus, the first case is the most favorable to a signal balance, on the one hand, and to the increase of the levels, on the other hand.

In total, if we consider that we have: $. a_{31}<a_{32} . a_{21}<a_{22} . a_{11}<a_{12} . a_{21}<a_{11}$ and $. a_{32}<a_{22}$, the result (I) and the first case, give the following balance:

$a_{31}<a_{32}<a_{2} 1<a_{22}<a_{11}<a_{12}$

\subsection{Subsidy policy}

If public policy breaks away from any principle of differentiation, and decides to allocate aid (monetary, selective, social and psychological) without any discrimination, the main obsession of which is to increase the level of basic training of the population.

We consider " $s$ " the amount of public subsidies distributed without any discrimination, we will have " $a_{11}-s=a_{21}$ ", to bring the agents of group " $A$ " (the base) to the level " $x *$ ", two cases present:

First case: $a_{11}-s=a_{21}$ and $a_{22}-s>a_{32}$ :

We will have a concentration in the level " $x * "$, and this level will take the place of level " $0 "$. As a result, the " $x * "$ level is depreciated, and the " $y^{* \prime \prime}$ level remains intact.

Second case: $a_{11}-s=a_{21}$ and $a_{22}-s=a_{32}$ :

There is a depreciation of the " $x *$ and $y^{* 1}$ level.

Public aid must therefore be directed towards the most productive individuals, who come from the least advantaged backgrounds (socially and geographically). However, the academic criterion must be protected from any intervention, and the targeted public aid must not have an impact on the relevance of this criterion.

Otherwise, the diploma loses its signaling capacity, and employers will increasingly seek high-level graduates, and the accumulation principle means that all diplomas will be affected by this depreciation. In this case, we will have a dramatic situation, where the agents will increase their training level as much as possible, monopolize the highest diplomas in a first stage, and lose all motivation for these diplomas in a second stage.

\section{Conclusion}

Reflection on training based on the theory of human capital is a fertile field of study still largely unexplored, but of which we increasingly measure the importance especially in research addressing solutions to fight unemployment, the phenomena of under and over qualification, or even the wage gaps.

The relationship at the heart of human capital theory illustrates the link between wages and education. However, this link is not sufficient to strictly demonstrate this relationship. In other words, it must be ensured that people who have completed longer studies necessarily receive higher incomes. Without this, human capital theory is powerless to explain the demand for education. The vast literature that has been produced on this subject is commensurate with this issue and its objective is to assess as accurately as possible the value of private returns to education. 
This must guide individual educational behavior and public policies.

The theory is however not systematically verified. In fact, other variables come into play that has nothing to do with productivity (economic context, specifics of the company, regions, structure of the labor market, sector of activity, gender or age of the worker....).

The filter theory initially presented by Arrow explains that training, and in particular the diploma, serves to provide information on the aptitudes of individuals (intelligence, work capacity, etc.). Education, therefore, does not serve to increase the productivity of individuals but to identify them so that they can be filtered. Arrow is interested in the costs and benefits to the community of such a screening process.

Signal theory is an extension of the job market of that of the filter.

Spence believes that education would not increase the agent's productivity, but would select the agents who are already and will be the most productive. In this case, it is necessary to question the social profitability of education which involves significant costs without improving worker productivity. The diploma obtained is therefore simply a signal for the employer, it is proof that the agent is better than the others and that he has been selected.

The reexamination of the filter theory in our study exposes the idea of strengthening the filtering function within universities, to reexamine the fall in the real costs of higher studies, and above all to regularize university studies with the model of Market.

Where we have differentiated four types of filters:

First, the perfect non-productive filter, in this case, we notice that there is a hypersensitivity to aptitude: if the aptitude is doubled within the population, the level of education will be multiplied by more than "4". This illustration is strictly in favor of the fittest.

Then, the imperfect non-productive filter where we distinguish two cases:

- Funding opportunities within the population exceed skills. In this case, we will see a new regulation of wages by employers.

- The volume of skills in the population exceeds the funding opportunities. In this case, individuals will try to invest more than necessary in education so that their skills are better distinguished.

Then, the productive and balanced filter the study shows that those of which they have higher aptitudes will seek the highest signal. And if we cancel the effect of education, the demand for the signal is reduced to the additional skill (the demand for the highest signals) and similarly, the difference in net income is limited only to the difference in skill.

Finally, the filter and job occupied, in this case, the analysis shows that individuals in the unfiltered sector spend less time in school than those in the filtered sector. However, the income distribution in the unfiltered sector is lower than that distributed in the filtered sector. However, these income supplements are greatly reduced.

In this study, we have also presented a reformulation of the Spence model by adopting three groups of individuals, and two levels of discrimination.

This, on the one hand, illustrates the possible choices and the relative gains of a holder of an average diploma. On the other hand, allow us to highlight three choices instead of two (medium signaling, strong signaling, and no signaling).

The study shows that the reporting process for middle-level diplomas is only valid when the number of individuals with higher-level diplomas does not exceed half of the least educated, which means that the signal effect does not own its own strengths, it depends on other signals.

In this regard, we explain the most favorable case for signal balance, on the one hand, and increasing training levels, on the other.

However, the formulations presented were based on strong hypotheses, supposing that there is a perfect match between skills, marginal productivity, and diploma, and also canceling market imperfections, which can present a limit to this study.

\section{References}

Acemoglu, D. (1999). Changes in Unemployment and Wage Inequality: An Alternative Theory and Some Evidence, American Economic Review, 89(5), pp. 1259-1278.

Arrow, J. (1973). Higher education as a filter. Journal of Public Economics. July; 1.

Ashenfelter, O.; Kruger, A. (1994). Estimates of the economic return to schooling from a new sample of twins, American Economic Review, 84, pp. 1157-1173.

Becker, G. Chiswick, B. (1966). Education and the distribution of earnings. American economic review. May.

Blanchard, O.; Diamond, P. (1994). Ranking, Unemployment Duration, and Wages, Review of Economic Studies, 6, pp. 417-434

Burdett, K.; Mortensen, D. (1998). Wage Differentials, Employer Size, and Unemployment, International Economic Review, 39(2), pp. 257-273, May.

Davidson, C.; Matusz, S.; Shevchenk, O. (2007). Globalization and firm level ad-justment with imperfect labor markets, Working paper, Michigan State University, Lansing. 
Gamel, C. (2000). Le diplôme, un signal en voie de dépréciation ? Le modèle de Spence réexaminé. Revue d'économie politique, 110(1), 53-84.

Gautier, P.;Van den Berg, G.; Van Ours, J.; G. Ridder, G. (2002). Worker turnover at the firm level and the crowding out of lower educated workers, European Economic Review, 46, pp. 523-538.

Hagedorn, M.; Manovskii, I. (2008). The Cyclical Behavior of Equilibrium Unemployment and Vacancies Revisited, American Economic Review, 98(4), pp. 1692-1706, September.

Moen, E. (1999). Education, Ranking, and Competition for Jobs, Journal of Labor Economics, 17(4), pp. 694-723.

Mortensen, D.; Nagypal, E. (2007). More on Unemployment and Vacancy Fluctuations, Review of Economic Dynamics, 10(3), pp. 327-347, July.

Mortensen, D.; Pissarides, C. (1999). New developments in models of search in the labor market, Handbook of Labor Economics, 3(39), pp. 2567-2627.

Noldeke, G.; Vandamme, E. (1990). Signalling in a dynamic labour market. Review of Economic Studies, 57, 123.

Pissarides, C. (2000). Equilibrium Unemployment Theory, 2nd Edition, MIT Press Books, The MIT Press, edition 1, Vol. 1, number 0262161877.

Postel-Vinay, F.; Robin, J. (2002). Equilibrium Wage Dispersion with Worker and Employer Heterogeneity, Econometrica.

Sahin, A.; Joseph, S.; Giorgio, T.;Giovanni, L. (2014). Mismatch Unemployment, American Economic Review, 104 (11), pp. 3529-64.

Shimer, R. (2012). Reassessing the Ins and Outs of Unemployment, Review of Economic Dynamics, 15(2), pp. $127-48$.

Spence M. Job market signaling. Quarterly journal of Economics. 1973 August; 87(3).

Spence M. Signaling in Retrospect and the Informational Structure of Markets. American Economic Review. 2002; $92,434-459$.

Yashiv, E. (2000). The Determinants of Equilibrium Unemployment, American Economic Review, 90(5), pp. 1297-1322, December. 